\title{
Preparation and Antioxidant Activity of Lycium barbarum Polysaccharide Iron Complex
}

\author{
Li-ming $\mathrm{JIN}^{\mathrm{a}}{ }^{*}$, Fang-ning GUO ${ }^{\mathrm{b}}$, Ai-li JIANG ${ }^{\mathrm{c}}$ and Wen-zhong $\mathrm{HU}^{\mathrm{d}}$ \\ College of Life Science, Dalian Nationalities University, Dalian 116600, China \\ aemail:jlm@dlnu.edu.cn, bemail:634992708@qq.com, cemail:jal@dlnu.edu.cn, \\ demail:hwz@dlnu.edu.cn \\ * corresponding author
}

Keywords: Lycium barbarum Polysaccharide Iron Complex(LBP-Fe), Synthesis, Characterization, Antioxidant activity

Abstract. The aim of the present research was to prepare an organic iron compound, Lycium barbarum polysaccharide iron complex (LBP-Fe), and evaluate its antioxidant abilities on scavenging super oxide radicals and DPPH radicals in vitro. LBP-Fe was prepared by chemically modification using chloride ferric and Lycium barbarum polysaccharide (LBP). The content of Fe was determined by flame atomic absorption spectrometry (FAAS) and the result was $36.71 \mathrm{mg} / \mathrm{g}$. LBP-Fe was characterized by ultraviolet (UV) and infrared (IR) techniques. The results proved the successful synthesis of LBP-Fe. The antioxidant activities on scavenging super oxide radicals and DPPH radicals of LBP-Fe were investigated by spectrophotometry. The results showed that in the setting concentration range, the antioxidant activities increased with the elevation of concentration. The antioxidant activities of LBP-Fe were better than that of LBP. The clearance rates on superoxide anion radicals were $51.39 \%$ for $2.0 \mathrm{mg} / \mathrm{mL} \mathrm{LBP}$ and $58.25 \%$ for $2.0 \mathrm{mg} / \mathrm{mL} \mathrm{LBP-Fe}$. The scavenging rates on DPPH radicals of $2.0 \mathrm{mg} / \mathrm{mL} \mathrm{LBP}$ and LBP-Fe were $45.23 \%$ and $48.76 \%$, respectively. The results suggest that LBP-Fe has potent antioxidant properties and could be applied as a novel iron source in dietary supplements.

\section{Introduction}

Lycium barbarum, also known as goji berry or wolfberry, is a kind of famous Chinese herbal medicine. It is beneficial to immune-stimulating, reducing fats, anti-aging and anti-tumor, therefore, it is popular in China, also has spread to subtropical regions from southeastern Asia to Europe and North America[1]. Lycium barbarum polysaccharide (LBP) is one of the main effective components.

Iron is an important mineral to the human for maintaining normal functions, such as oxygen transport, DNA synthesis, ATP production and other physiological processes[2]. The World Health Organization reported that about $46 \%$ of the world's 5- to 14-years-old children and $48 \%$ of the pregnant women were anemic, most of which were due to iron deficiency[3]. Ferrous sulfate was used as oral iron supplement for a long time in clinical practice, however, it could lead to adverse effects, including nausea, vomiting, irregular absorption and abdominal pain. Nowadays nontoxic polysaccharide-iron complexes become popular iron supplements to treat the iron-deficiency anemia with no or little side effect[4].

The objective of this study was to prepare Lycium barbarum polysaccharide iron complex (LBP-Fe) and evaluate its antioxidant abilities, including scavenging effects on super oxide radicals and DPPH radicals.

\section{Materials and methods}

Materials. Lycium barbarum polysaccharide (LBP) crude extract was purchased from XiAn TianYi Biotechnology Co. 1,1-diphenyl-2-picrylhydrazyl (DPPH), Nitro Blue tetrazolium(NBT), nicotinamide adenine dinucleotide-reduced(NADH), phenazine methosulfate(PMS), were purchased from Sigma Chemical Co. All other analytical grade chemicals used were obtained from Tianjin Kemio Chemical Co. 
Purification of LBP. The crude LBP was deproteined according to the Sevage method. Briefly, $10.0 \mathrm{~g}$ LBP extract was dissolved in $100 \mathrm{~mL}$ deionized water. The solution was treated with sevage reagent (1-butanol:chloroform=1:4) and then fully oscillated for $20 \mathrm{~min}$. After centrifuged $4000 \mathrm{r} / \mathrm{min}$ for $10 \mathrm{~min}$, the superstratum solution was remained. The procedure above was repeated until the supernatant was no free protein. The mixture was added into ethanol $(1: 3, \mathrm{~V}: \mathrm{V})$ to be precipitated at $4^{\circ} \mathrm{C}$ over night. Finally, the precipitate was filtrated and lyophilized. Total sugar content of purified LBP was quantified by phenol-sulphuric acid method with glucose as the standard.

Synthesis of LBP-Fe. $0.5 \mathrm{~g}$ purified LBP was dissolved in $50 \mathrm{~mL}$ deionized water and mixed with $0.5 \mathrm{~mL} 1 \%$ acetic acid. The mixed solution was stirred and then added into $0.65 \mathrm{~g}$ chloride ferric, which was dissolved in $10 \mathrm{~mL}$ deionized water. After $3 \mathrm{~h}$ at room temperature, $3 \mathrm{~V}$ ethanol was added in order to be precipitated overnight. The precipitate was centrifugated and washed twice with ethanol. After dialysis, the production LBP-Fe was obtained by lyophilization.

Property and Characterization of LBP-Fe. UV spectra were measured by a Lambda 25 UV-VIS spectrophotometer. IR spectra were obatained by a 370 DTGS IR spectrometer with $\mathrm{KBr}$ disks. The content of Fe was determined by a Z-2000 flame atomic absorption spectrometry (FAAS).

Scavenging activity on super oxide radicals and DPPH radicals. The evaluation method of scavenging activity on super oxide radicals and DPPH radicals was according to Wang et al[4] and Jin et al[5].

\section{Results and Discussion}

Purification of LBP. Through phenol-sulphuric acid method and glucose standard curve, total sugar content of LBP was quantified of $78.95 \%$.

Synthesis of LBP-Fe. LBP-Fe prepared was red brown powder. The yield was $39.75 \%$ and the content of Fe was $36.71 \mathrm{mg} / \mathrm{g}$.

Structure analysis. The UV spectrum (Fig.1) showed that the optimum absorption peaks of LBP were $224 \mathrm{~nm}$ and $282 \mathrm{~nm}$, respectively. However, the two optimum absorption peaks disappeared in LBP-Fe spectrum. The differences confirmed the structure changes between LBP and LBP-Fe. In the IR spectrum (as shown in Fig.2), hydroxyl stretching vibration absorption peak shifted from $3346 \mathrm{~cm}^{-1}$ (LBP) to $3421 \mathrm{~cm}^{-1}$ (LBP-Fe). In addition, two new absorption peaks of $1400 \mathrm{~cm}^{-1}$ and $1701 \mathrm{~cm}^{-1}$ were observed in LBP-Fe IR spectrum. According to the results, chloride ferric was probably linked to hydroxyl group.

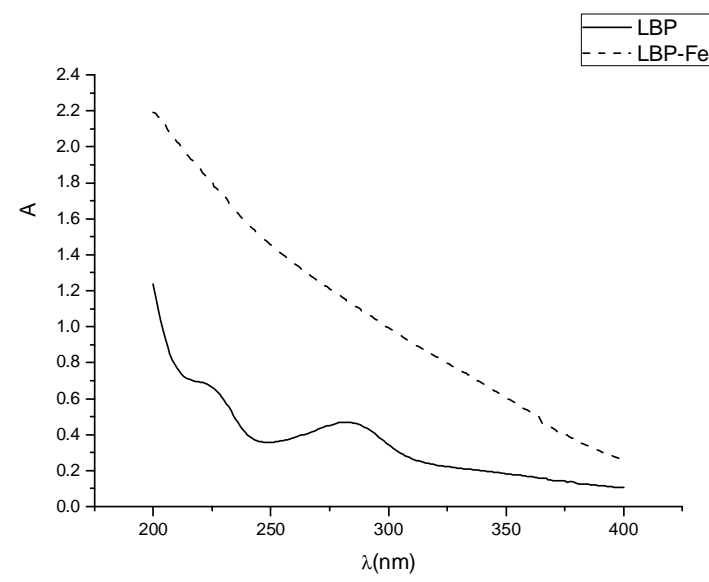

Fig.1 UV Spectrum of LBP and LBP-Fe 


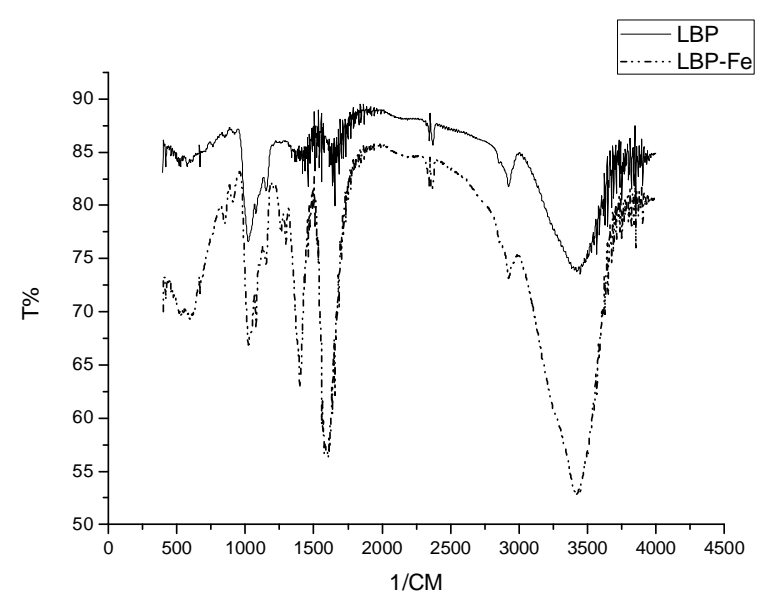

Fig.2 IR Spectrum of LBP and SeLBP

Scavenging activity of superoxide radicals. As shown in Fig.3, the inhibiting activities of LBP-Fe and LBP on superoxide radicals were concentration related and the effect of LBP-Fe was better than LBP. The scavenging effects of $2.0 \mathrm{mg} / \mathrm{mL} \mathrm{LBP}$ and LBP-Fe were $51.39 \%$ and $58.25 \%$, respectively, which were higher than $\mathrm{V}_{\mathrm{C}}$.

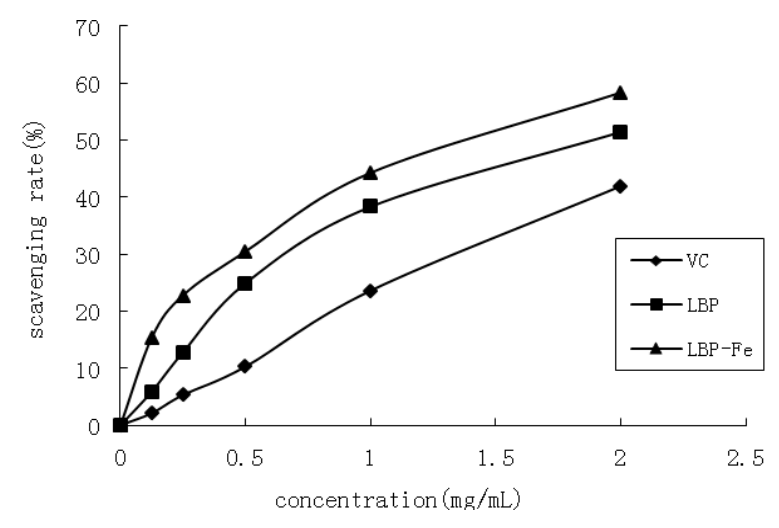

Fig. 3 Scavenging effect of LBP, LBP-Fe and VC on superoxide radicals

Scavenging activity of DPPH radicals. As seen in Fig.4, the scavenging rates of LBP-Fe and LBP increased with increasing concentration from $0.125 \mathrm{mg} / \mathrm{mL}$ to $2.0 \mathrm{mg} / \mathrm{mL}$. LBP-Fe showed better scavenging ability on DPPH radicals than LBP. At the concentration of $2.0 \mathrm{mg} / \mathrm{mL}$, the scavenging rate to DPPH radicals of LBP-Fe and LBP were $48.76 \%$ and $45.23 \%$, respectively, which were little higher than Vc. This was similar to the results of scavenging activities on superoxide radicals.

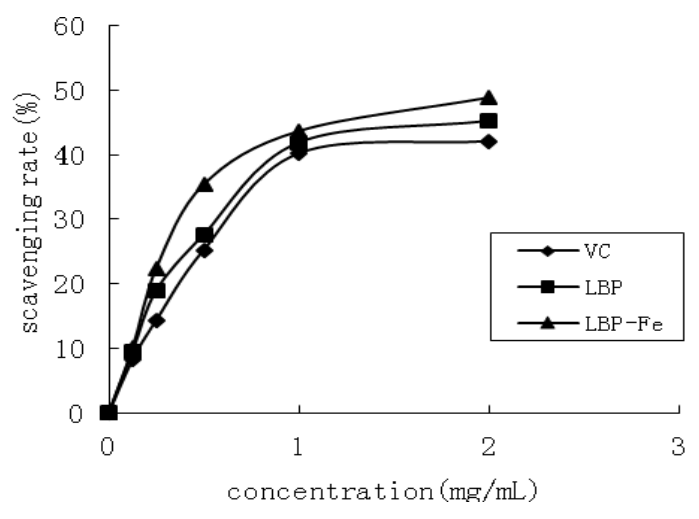

Fig. 4 Scavenging effect of LBP, LBP-Fe and $V_{C}$ on DPPH radicals 


\section{Conclusion}

In this paper, a kind of organic polysaccharide iron complex, LBP-Fe, was synthesized successfully. It showed the concentration related scavenging activities on superoxide radicals and DPPH radicals which were better than that of LBP. It was probably due to the introduction of iron. It is well known that iron plays crucial roles in oxygen transport, furthermore, iron ions can catalyze the formation of the hydroxyl radical and accelerate the decomposition of lipid hydroperoxides. Diet is the main source of iron for population. LBP-Fe could potentially serve as a low toxic and low cost dietary supplement of organic iron with antioxidant activity.

\section{Acknowledgment}

This work was financially supported by the National Science and Technology Support Program (Grant No. 2012BAD38B05), Fund of Education Department of Liaoning Province(L2013510), Fundamental Research Fund for the Central Universities (DC201501020302, DC201501020101), Innovation and Entrepreneurship Training Program for College Students of Dalian Nationalites University (X201503068).

\section{References}

[1] M. Forino, L. Tartaglione, C. D. Aversano, P. Ciminiello, NMR-based identification of the phenolic profile of fruits of Lycium barbarum (goji berries). Isolation and structural determination of a novel $\mathrm{N}$-feruloyl tyramine dimer as the most abundant antioxidant polyphenol of goji berries, Food Chem. 194 (2016) 1254-1259.

[2] M.M. Tang, D.F. Wang, Y.F. Hou, P. Buchili, L.P. Sun, Preparation, characterization, bioavailability in vitro and in vivo of tea polysaccharides-iron complex, Eur. Food Res. Technol. 236 (2013) 341-350.

[3] H. Tapiero, L. Gaté, K.D. Tew, Iron: deficiencies and requirements, Biomed. Pharmacother. 55 (2001) 324-332.

[4] J. Wang, H.X. Chen, Y.W. Wang, L.S. Xing. Synthesis and characterization of a new Inonotus obliquus polysaccharide-iron(III) complex, Inter. J. Bio. Macromol. 75 (2015) 210-217.

[5] L.M. Jin, N. Geng, C.S. Quan, S.D. Fan, Synthesis and antioxidant activity of carboxymethyl chitosan-selenium, Advanced Materials Research.734-737 (2013) 2100-2103. 